\title{
Corrections to: The Trauma Golden Hour
}

Adonis Nasr, Flavio Saavedra Tomasich, Iwan Collaço, Phillipe Abreu, Nicholas Namias, and Antonio Marttos

\section{Correction to:}

A. Nasr et al. (eds.), The Trauma Golden Hour, https://doi.org/10.1007/978-3-030-26443-7

The affiliation of co-editors Nicholas Namias and Antonio Marttos was incorrectly updated in the book front matter and this is corrected as follows:

Ryder Trauma Center

Jackson Memorial Hospital

University of Miami

Miami, FL

USA 